\title{
The interior of the SNR RX J0852.0-4622 (Vela Jr) at radio wavelengths
}

\author{
E. M. Reynoso ${ }^{1,2, \star}$, G. Dubner ${ }^{1, \star}$, E. Giacani ${ }^{1, \star}$, S. Johnston ${ }^{3,2}$, and A. J. Green ${ }^{2}$ \\ 1 Instituto de Astronomía y Física del Espacio (IAFE), CC 67, Suc. 28, 1428 Buenos Aires, Argentina \\ e-mail: ereynoso@iafe.uba.ar \\ 2 School of Physics, University of Sydney, NSW 2006, Australia \\ 3 Australia Telescope National Facility, Commonwealth Scientific and Industrial Research Organisation, Post Office Box 76, Epping, \\ NSW 1710, Australia
}

Received 22 September 2005 / Accepted 23 November 2005

\section{ABSTRACT}

Aims. We observed the center of the supernova remnant Vela Jr in radio continuum in order to search for a counterpart to the compact central X-ray source CXOU J085201.4-461753, possibly a neutron star candidate which could be the remnant of the supernova explosion. Methods. Observations were made with the Australia Telescope Compact Array at 13 and $20 \mathrm{~cm}$. Spectral indices were obtained using flux density correlations of the data which were spatially filtered to have the same $u-v$ coverage. A multiwavelength search for counterparts to the compact central X-ray source was made.

Results. We compiled a new catalogue of 31 small diameter radio sources, including the previously known source PMN J0853-4620, listing the integrated flux densities at $20 \mathrm{~cm}$ and, for half of the sources, the flux densities at $13 \mathrm{~cm}$ with the corresponding spectral indices. All sources are unresolved at the present angular resolution except for Source 18, which is clearly elongated and lies strikingly close to CXOU J085201.4-461753. Our observations show no evidence for the existence of a pulsar wind driven nebula associated with the point X-ray source. Furthermore, Source 18 has a thermal spectrum with index $\alpha=+0.8 \pm 0.4\left(S \propto v^{\alpha}\right)$, and appears to be the counterpart of the optical source Wray 16-30. In spite of the absence of [O III] emission lines as reported in the literature, we find that this object could be explained as a low emission planetary nebula belonging to the "butterfly" morphological class.

Conclusions. We conclude that if the radio source 18 is actually a planetary nebula, then CXOU J085201.4-461753 is more likely to be related to it rather than to Vela Jr.

Key words. ISM: supernova remnants - ISM: individual objects: Vela Jr - ISM: planetary nebulae: individual: Ve 2-27 stars: individual: Wray 16-30 - X-rays: individual: CXOU J085201.4-461753 - catalogs

\section{Introduction}

Rotation powered pulsars can lose their energy in the form of a wind of electrons and positrons, creating a synchrotron emitting nebula or plerion, generally called a "pulsar wind nebula" (PWN). In the last few years, new data with unprecedented spatial resolution have revealed that PWNe are highly structured objects which can fall into a variety of morphological classes depending on the properties and evolutionary state of the pulsar and its surroundings (Gaensler 2004). The morphology of a PWN depends on how the wind particles are confined. Therefore, the study of PWNe are of particular interest since their properties can provide information on both, the pulsar's wind and the surrounding interstellar medium.

In some cases, neutron stars (NS) are not detected as ordinary radio pulsars. Several X-ray sources with no radio

^ Member of the Carrera del Investigador Científico, CONICET, Argentina. counterpart and very high X-ray to optical flux ratios have been detected projected on the interior of supernova remnants (SNR). These sources, generally called "compact central objects" (CCO; see Pavlov et al. 2001), are believed to be NS with peculiar characteristics (e.g. Frail 1998; Geppert et al. 1999), or simply normal pulsars with unfavorable beaming (Brazier \& Johnston 1999). In recent papers, we have reported the results of an $\mathrm{H} \mathrm{I}$ and radio continuum survey towards candidate NSs to search for their imprints in the interstellar medium and confirm or reject their physical association with the host SNR (Reynoso et al. 2003, 2004a; Giacani et al. 2005). In this paper we analyze another CCO-SNR possible association: the X-ray source CXOU J085201.4-461753 and the SNR Vela Jr.

Vela Jr (G266.1-1.2, RX J0852.0-4622) is a shell-like $\mathrm{SNR}$, about $2^{\circ}$ in diameter, first discovered with ROSAT (Aschenbach 1998). The name "Vela Jr" is due to its position at the south-east corner of the Vela SNR, and to a possible age of $\sim 700 \mathrm{yr}$ based on the detection of $\gamma$-ray emission from the 
radioactive isotope ${ }^{44} \mathrm{Ti}$ (Iyudin et al. 1998). However, subsequent observations show that this source is likely to be older and ten times more distant $(2 \mathrm{kpc})$ than was originally believed (Slane et al. 2001). Combi et al. (1999) reported a radio counterpart based on the $2.42 \mathrm{GHz}$ survey of Duncan et al. (1995). More recently, Duncan \& Green (2000) find that several features believed to be extensions to the radio shell are actually unrelated to Vela Jr. A detection with HESS of TeV $\gamma$-ray extended emission, whose spatial distribution correlates with the X-ray observations, has recently been reported (Aharonian et al. 2005).

Several authors noted the presence of an X-ray point-like source close to the SNR center: Aschenbach et al. (1999) based on the ROSAT All Sky Survey, Pavlov et al. (2001) using the Chandra Spectrometer, Slane et al. (2001) based on ASCA data, and Mereghetti (2001) with BeppoSAX observations. This point-like source is named slightly differently according to the different authors and has independent entries listed in SIMBAD. Most frequently, it is referred to by its Chandra identification CXOU J085201.4-461753. Due to its location, this X-ray source is believed to be the NS remaining after the supernova explosion. This identification of CXOU J085201.4-461753 as a NS appears to be supported by the very high X-ray-to-optical flux ratio (Pavlov et al. 2001). Although the distance to CXOU J085201.4-461753 is poorly known, if it is located at $2 \mathrm{kpc}$ the emission region and luminosity are similar to the CCO detected in Cas A. Pellizzoni et al. (2002) found a faint $\mathrm{H} \alpha$ nebula at a position compatible with CXOU J085201.4-461753, which could be explained as a bow shock driven by a NS. However, Iyudin et al. (2005) claim that the very high ejecta velocity derived for Ti implies that the $\mathrm{SN}$ was of type Ia, in which case no compact remnant is expected. The nature of CXOU J085201.4-461753 thus remains uncertain.

Reynoso et al. (2004b) observed a $\sim 30^{\prime}$ diameter field towards CXOU J085201.4-461753 with the Australia Telescope Compact Array ${ }^{1}$ (ATCA; Frater et al. 1992) in the $\lambda 21 \mathrm{~cm}$ line and at $20 \mathrm{~cm}$ in the radio continuum. The observations revealed a compact continuum source and an elongated region $\left(30^{\prime} \times 14^{\prime}\right)$ of enhanced emission at the position of the $\mathrm{X}$ ray source. If confirmed, this could be a PWN and would be good evidence that the compact X-ray source is a neutron star. However, this enhancement is only marginally above the noise level of the image (less than $3 \sigma \mathrm{rms}$ ), making the detection of the nebula doubtful. In this paper, we analyze new observations of the central region of Vela Jr performed at 13 and $20 \mathrm{~cm}$, which confirm the detection of the compact source. A new interpretation of the source origin is presented.

\section{Observations and data reduction}

The new observations were obtained with the ATCA, the 22-m six antenna array located in Narrabri, NSW, Australia. To optimize the $u-v$ coverage, the observations were carried out in 2004 during two sessions of $12 \mathrm{~h}$ each, on February 19 in the

\footnotetext{
${ }^{1}$ The ATCA is part of the Australia Telescope, which is funded by the Commonwealth of Australia for operation as a National Facility managed by CSIRO.
}

750A array configuration and on April 9 in the EW367 configuration. In these two configurations, the antennas are aligned in the EW direction, and the baseline lengths given by the five movable antennas vary from 30 to $735 \mathrm{~m}$. The shortest baseline and the weighting scheme used set an upper limit for the size of well imaged structures, estimated to be $\sim 35^{\prime}$ at $20 \mathrm{~cm}$ and $\sim 24^{\prime}$ at $13 \mathrm{~cm}$, although mosaicing helps to partially recover short spacings and thus increase these limits. The inclusion of the sixth antenna, fixed in location $3 \mathrm{~km}$ away from the remainder of the array, increases the $u-v$ coverage by the addition of baselines from $\sim 3000$ to $4440 \mathrm{~m}$. The observations were made in continuum mode, which allows the simultaneous measurement of two frequency bands, centred on $1384 \mathrm{MHz}$ and $2368 \mathrm{MHz}$, each with a correlator bandwidth of $128 \mathrm{MHz}$.

The dimensions of the region of enhanced emission found in Reynoso et al. (2004b), required that the observations be made in mosaicing mode with six pointings on a hexagonal grid chosen to preserve Nyquist sampling. A seventh pointing was observed regularly towards the bright $\mathrm{H}$ II region RCW 38, located $\sim 2^{\circ}$ away, to assist modelling of the sidelobes from this source in the data reduction. The source PKS 0823-500 was observed once per hour to calibrate the phases, while PKS 1934-638 was used for flux density and bandpass calibration. Each pointing of the mosaic was reduced individually with the MIRIAD software package (Sault et al. 1995), and the resulting images were combined as a mosaic using the MIRIAD task LINMOS, which automatically applies a primary beam correction. The $13 \mathrm{~cm}$ images were improved by restricting the uvrange to less than $18 \mathrm{k} \lambda$. The spatial resolution of the finished datasets is $37^{\prime \prime} .4 \times 31^{\prime \prime} .7$, at position angle $(\mathrm{PA})=-3.4$ for the $20 \mathrm{~cm}$ image, and $36.6^{\prime} 5 \times 26^{\prime \prime} 3$, at $\mathrm{PA}=-1.7$ for the $13 \mathrm{~cm}$ image. The sensitivities achieved are 0.2 and $0.1 \mathrm{mJy}^{\text {beam }}{ }^{-1}$ respectively.

\section{Results}

Figure 1 shows the emission at $20 \mathrm{~cm}$ in the central region of Vela Jr. A number of compact sources can be seen in the field, together with several filaments of faint emission. Some of the extended emission is confused by sidelobes from RCW 38 that could not be removed. To analyze to what extent the extended emission in Fig. 1 is reliable, four contour levels have been plotted on the grayscale image at $3 \sigma$ intervals, starting from $3 \sigma$. Most of the emission appears to be of very low significance, except for the filament that extends from Dec $\simeq-46^{\circ} 15^{\prime}$ to $-46^{\circ} 40^{\prime}$, at $\mathrm{RA} \simeq 8^{\mathrm{h}} 50^{\mathrm{m}} 15^{\mathrm{s}}$. The sidelobes around this feature are indicative that strong emission is present. Other features that appear genuine are those centered at about RA = $8^{\mathrm{h}} 49^{\mathrm{m}} 50^{\mathrm{s}}$, Dec $=-46^{\circ} 8^{\prime}$ and RA $=8^{\mathrm{h}} 51^{\mathrm{m}} 40^{\mathrm{s}}$, Dec $=-46^{\circ} 40^{\prime}$ and possibly a second filament parallel to the brightest one, at $\mathrm{RA} \simeq 8^{\mathrm{h}} 48^{\mathrm{m}} 40^{\mathrm{s}}$. The apparent curvature to the west of the bright filaments detected on the western half of the image suggests a probable connection with the extended Vela SNR.

At $13 \mathrm{~cm}$, the extended emission, if any, cannot be disentangled from the background noise level, but half of the compact sources still appear. The small source reported in Reynoso et al. (2004b) at a position compatible with CXOU J085201.4-461753 appears at both frequencies as a small, faint, 


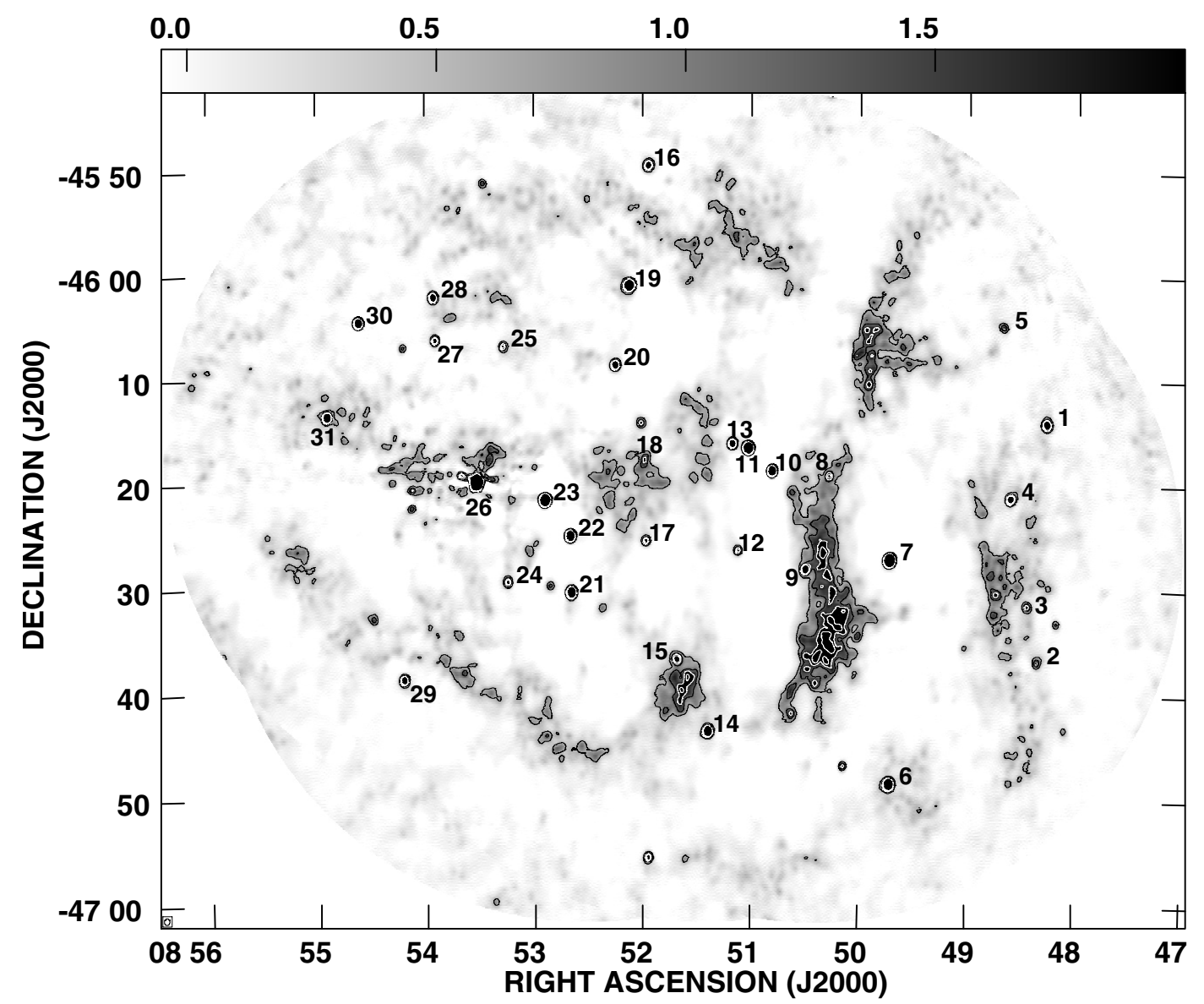

Fig. 1. Radio continuum image of G266.1-1.2 at $1384 \mathrm{MHz}$. The grayscale is given on top of the image in mJy beam ${ }^{-1}$. The contours are plotted at $3 \sigma, 6 \sigma, 9 \sigma$, and $12 \sigma$, where $\sigma=0.2 \mathrm{mJy}_{\text {beam }}{ }^{-1}$. The numbers correspond to the compact sources listed in Table 1 . The beam is plotted at the bottom left corner.

elliptical feature. In contrast with other similar features in the field, this source is resolved. After deconvolution, it has a size of about $130^{\prime \prime} \times 50^{\prime \prime}$ at a position angle (measured from $\mathrm{N}$ to $\mathrm{E}$ ) of $170^{\circ}$ at $20 \mathrm{~cm}$, and $50^{\prime \prime} \times 15^{\prime \prime}$ at a position angle of $150^{\circ}$ at $13 \mathrm{~cm}$. Figure 2 shows a close up of a region around this source at $20 \mathrm{~cm}$. The symbols correspond to the sources listed in Sect. 3.2 .

\subsection{Small-diameter sources in the field}

To investigate the nature of the compact sources appearing in the field, we estimated their positions and fluxes at both frequencies and calculated the spectral index between 13 and $20 \mathrm{~cm}$ where possible. The results are summarized in Table 1. The coordinates were obtained from a Gaussian fit to the flux density peak. When necessary, fluxes were corrected for contamination from background extended emission; this effect is more significant at $20 \mathrm{~cm}$. The spectral indices were derived for each source from the comparison of the two images using the flux-flux plotting technique (Costain 1960; Turtle et al. 1962). This method compares the flux densities at two frequencies within a selected region of the sky for which the data have been spatially filtered for the same $u-v$ coverage. A linear fit to the plot gives a measure of the spectral index. This method

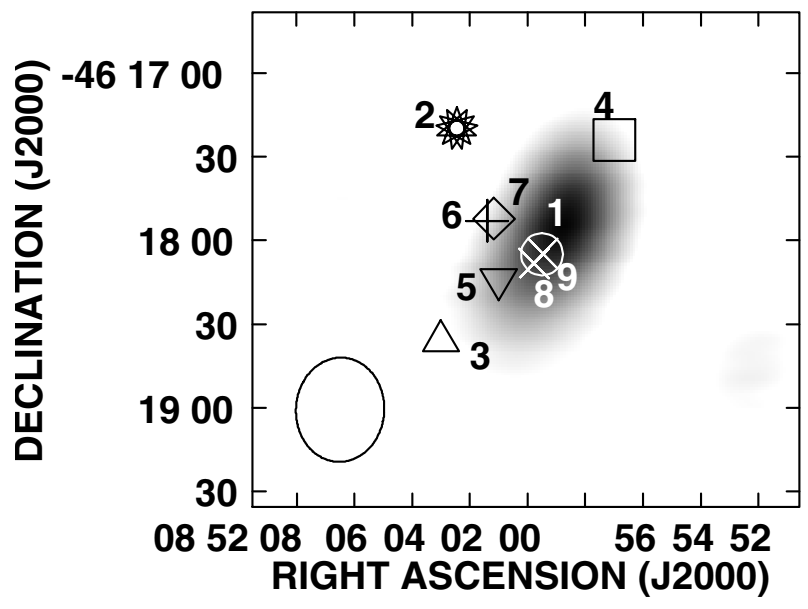

Fig. 2. Close up of a $\sim 4^{\prime} \times 4^{\prime}$ region around Source 18 (Table 1) at $1384 \mathrm{MHz}$. The beam is plotted at the bottom left corner. The symbols are as follows: circle, Wray 16-30; star, HD 76060; triangle, J085203-461836; square, AX J0851.9-4617; inverted triangle, J085201-461814; plus sign, CXOU J085201.4-461753; diamond, EQ J0852-4617; and crosses, MSX G266.2348-01.2004 and IRAS 0852-4606. The numbers correspond to the sources listed in Table 2.

is useful because it is unaffected by absolute calibration and offset variations between the images. 
Table 1. Small-diamter sources in the field.

\begin{tabular}{|c|c|c|c|c|c|}
\hline & $\begin{array}{c}\text { RA (J2000) } \\
\text { h m s s }\end{array}$ & $\begin{array}{c}\operatorname{Dec}(\mathrm{J} 2000) \\
{ }^{\prime}, "\end{array}$ & $\begin{array}{l}S_{13 \mathrm{~cm}} \\
(\mathrm{mJy})\end{array}$ & $\begin{array}{l}S_{20 \mathrm{~cm}} \\
(\mathrm{mJy})\end{array}$ & $\begin{array}{c}\text { Spectral } \\
\text { index }\end{array}$ \\
\hline 1 & 084816.2 & -461411 & & 8.4 & \\
\hline 2 & 084820.9 & -463657 & & 2.0 & \\
\hline 3 & 084826.5 & -463135 & & 2.8 & \\
\hline 4 & 084836.0 & -462122 & & 6.7 & \\
\hline 5 & 084840.4 & -460458 & & 2.8 & \\
\hline 6 & 084942.7 & -464836 & & 20 & \\
\hline 7 & 084942.8 & -462719 & 28 & 49 & $-0.98 \pm 0.04$ \\
\hline 8 & 085016.8 & -461922 & 1.8 & 2.1 & $-0.35 \pm 0.10$ \\
\hline 9 & 085029.9 & -462817 & 4.2 & 5.0 & $-0.6 \pm 0.2$ \\
\hline 10 & 085048.2 & -461854 & 8.3 & 15 & $-1.2 \pm 0.1$ \\
\hline 11 & 085101.4 & -461643 & 33 & 31 & $0.21 \pm 0.02$ \\
\hline 12 & 085107.1 & -462630 & 3.5 & 3.2 & $0.12 \pm 0.13$ \\
\hline 13 & 085110.4 & -461617 & 4.9 & 8.0 & $-0.7 \pm 0.2$ \\
\hline 14 & 085123.6 & -464343 & 2.3 & 16 & \\
\hline 15 & 085141.0 & -463651 & & 4.6 & \\
\hline 16 & 085156.8 & -454937 & & 7.1 & \\
\hline 17 & 085158.1 & -462535 & 3.6 & 3.1 & $0.23 \pm 0.07$ \\
\hline 18 & 085158.9 & -461752 & 3.4 & 2.4 & $0.8 \pm 0.4$ \\
\hline 19 & 085207.3 & -460116 & 2.7 & 19 & \\
\hline 20 & 085215.1 & -460848 & 9.7 & 8.1 & $0.35 \pm 0.01$ \\
\hline 21 & 085239.5 & -463030 & 10 & 18 & $-0.95 \pm 0.07$ \\
\hline 22 & 085240.1 & -462507 & 12 & 17 & $-0.54 \pm 0.05$ \\
\hline 23 & 085254.1 & -462142 & 23 & 39 & $-0.92 \pm 0.04$ \\
\hline 24 & 085314.6 & -462936 & 2.1 & 3.9 & $-1.1 \pm 0.3$ \\
\hline 25 & 085317.1 & -460659 & & 2.8 & \\
\hline 26 & 085331.9 & -461959 & 192 & 226 & $-0.34 \pm 0.02$ \\
\hline 27 & 085354.7 & -460621 & & 3.8 & \\
\hline 28 & 085355.6 & -460215 & & 7.4 & \\
\hline 29 & 085412.6 & -463845 & & 7.1 & \\
\hline 30 & 085436.9 & -460438 & & 13 & \\
\hline 31 & 085454.6 & -461338 & & 10 & \\
\hline
\end{tabular}

To apply the flux-flux plots method, the image at $13 \mathrm{~cm}$ was convolved to the $20 \mathrm{~cm}$ beam size. To allow for poor correlation coefficients, the regression was done twice, by plotting data at $13 \mathrm{~cm}$ vs. $20 \mathrm{~cm}$ and vice versa. The spectral indices shown are the average of both estimates. The correlation coefficient was nearly one for almost all the sources. The quoted errors include a contribution from the intrinsic flux density errors plus the statistical error from the linear regression. Estimates were made only for sources with a flux density $>1 \mathrm{mJy} \mathrm{beam}^{-1}$ that were at least $3 \sigma$ above the image noise level. As an example, flux-flux plots for two representative sources (18 and 26) are shown in Fig. 3.

For sources 14 and 19, we do not list the spectral indices because they lie very close to the boundary of the calibrated image at $13 \mathrm{~cm}$, and their flux densities are incompletely recovered, which produces unrealistic values for the spectral index.

After searching the available published radio catalogs, we find that the only compact source previously identified in this field is number 26 in Table 1, listed in the Parkes-MIT-NRAO Survey (Wright et al. 1994) as PMN J0853-4620, with a flux density of $151 \mathrm{mJy}$ at $4.85 \mathrm{GHz}$. The spectral index derived for this source, including the PMN data, yields exactly the same result as using the flux-flux plot, giving us confidence in the reliability of the technique.

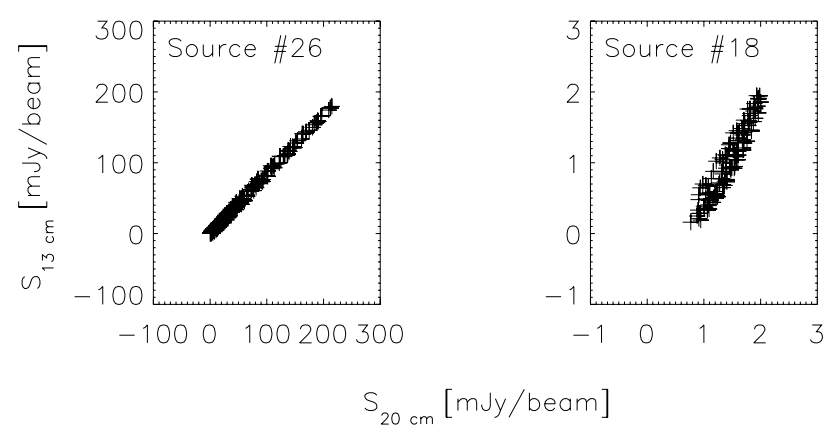

Fig. 3. Flux-flux plots for sources 18 (right) and 26 (left), where the flux density at $13 \mathrm{~cm}$ is plotted against the flux density at $20 \mathrm{~cm}$. Units are $\mathrm{mJy}_{\text {beam }}{ }^{-1}$.

The region observed for this paper has been partially imaged in the Molonglo Synthesis Telescope Galactic Plane Survey at $843 \mathrm{MHz}$ with sub arcmin resolution (Green et al. 1999). The sources and extended structures are consistent with the present data. Two images have also been published by Stupar et al. (2005) at 13 and $20 \mathrm{~cm}$, which are less sensitive but in broad agreement.

About half of the sources for which spectral indices have been computed appear to be extragalactic objects ( $\alpha$ more negative than -0.9$)$. The remaining sources have spectral indices comprised between $\sim-0.7$ and +0.8 . Source 18 is the only resolved source and is the target of our interest because of its proximity to CXOU J085201.4-461753. It has the highest positive spectral index in the field and we now discuss the possible origin of the emission associated with this source.

\subsection{Multi-wavelength data possibly related to the central source}

We have searched in the SIMBAD Astronomical Database for sources of all categories located at or close to the position of the radio nebula, Source 18. At least five X-ray sources, four optical objects and one radio source, are listed within a $1^{\prime}$ radius circle centered at the position $08^{\mathrm{h}} 52^{\mathrm{m}} 00^{\mathrm{s}} .0,-46^{\circ} 17^{\prime} 54^{\prime \prime}$. However, there are inconsistencies and possible duplications in the classifications from the published data. In an attempt to clarify the nomenclature we have listed in Table 2 the catalogued objects with their original names and positions as published by the respective authors, following in more or less historical order. We have excluded those sources labeled in SIMBAD as SNR RXJ 0852.0-4622, GRO J0852-4642, and [CRB99] A, which correspond to the X-ray, $\gamma$-ray, and radio manifestations of the SNR Vela Jr. A summary of these findings follows.

(1) Wray 16-30 was originally listed by Wray (1966) as a Be star, with coordinates accurate to about $\pm 15^{\prime \prime}$. This source is also listed in the SIMBAD database as Ve 2-27, an object originally classified as a planetary nebula (PN) with star-like appearance by Velghe (1957), and later as a peculiar Be star with infrared excess (Allen \& Glass 1975). In the StrasbourgESO Catalogue of Planetary Nebulae (Acker et al. 1992), it is classified as a peculiar emission-line source (see Landaberry et al. 2001, for a summary of the successive classifications of 
Table 2. Multi-wavelength detections near Source 18.

\begin{tabular}{llllll}
\hline \hline Number & Identifier & RA (J2000) & Dec (J2000) & Other names & References \\
& & h m s & & \\
\hline 1 & Wray 16-30 & 085159.5 & -461805 & ESO 260-PN11, Hen 2-14, & $1,2,3$ \\
& & & & Ve 2-27, PK 266-01 1, SS73 11 & 4 \\
2 & HD 76060 & 085202.45 & -461719.8 & & 5 \\
3 & [AIS99] J085203-461836 & 085203 & -461836 & 6 \\
4 & AX J0851.9-4617 & 085157 & -4617.4 & 7 \\
5 & [M2001b] J085201-461814 & 085201 & -461814 & 8 \\
6 & CXOU J085201.4-461753 & 085201.4 & -461753 & 8 \\
7 & EQ J0852-4617 & 085201.17 & -461752.3 & \\
8 & MSX G266.2348-01.2004 & 085159.8 & -46188.28 & & 10 \\
9 & IRAS 08502-4606 & 085159.5 & -46184.68 & & 9 \\
\hline
\end{tabular}

References: (1) Velghe (1957); (2) Landaberry et al. (2001) and references therein; (3) Wray (1966); (4) Hipparcos Catalogue, Perryman et al. (1997); (5) Aschenbach et al. (1999); (6) Slane et al. (2001); (7) Mereghetti (2001); (8) Pavlov et al. (2001); (9) MSX6C Infrared Point Source Catalog Egan et al. (1997); (10) IRAS Point Source Catalogue.

this emission-line object). Landaberry et al. (2001) find that Ve 2-27 (or Wray 16-30) is a peculiar emission-line star with a spectrum remarkably similar to that of the Luminous Blue Variable object $\eta$ Carinae, but with an unclear evolutionary status. However, according to the spectral study carried out by Landaberry et al. (2001), it is still possible to explain the observed spectrum as originating from a proto-planetary nebula.

(2) HD 76060 is catalogued in the Hipparcos Catalogue as a bright $(B=7.8) \mathrm{B} 8 \mathrm{IV} / \mathrm{V}$ star.

(3) [AIS99] J0825203-461836 refers to a point-like source discovered in the ROSAT all sky survey data by Aschenbach et al. (1999). It is about $3^{\prime} .4$ offset from the center of Vela Jr. A second point-like X-ray source is reported near $08^{\mathrm{h}} 51^{\mathrm{m}} 58^{\mathrm{s}}$, $-46^{\circ} 21^{\prime} 33^{\prime \prime}$. The authors discuss the possiblity that one of these sources could be a neutron star. However, since no spectra were available at that time, the analysis was incomplete.

(4) AX J0851.9-4617 was detected with ASCA as an unresolved X-ray source surrounded by diffuse emission and located near the center of SNR RXJ 0852.0-4622 (Slane et al. 2001). According to the authors, the properties of the source are not well determined but appear to be consistent with a neutron star surrounded by a synchrotron nebula, although an association with one of two stars located within the positional error circle is also possible.

(5) [M2001b] J085201-461814 is the X-ray source that Mereghetti (2001) reported to be at the center of the SNR RX J0852.0-4622. It should be noted that at energies $E \geq 5 \mathrm{keV}$ a new source was detected, indentified as SAX J0852.0-4617, which has a harder X-ray spectrum and higher X-ray-to-optical flux ratio than was measured for the original neutron star candidate AX J0851.9-4617. This new source is a more likely candidate for the central neutron star.

(6) CXOU J085201.4-461753 is a point X-ray source discovered with the Advanced CCD Imaging Spectrometer detector aboard Chandra. The source lies about 4 ' north of the center of the SNR RXJ 0852.0-4622. Pavlov et al. (2001) proposed that it is the compact remnant of the $\mathrm{SN}$ explosion.
(7) EQ J0852-4617 is a faint $\left(B \sim 19^{\mathrm{m}}\right)$ star reported by Pavlov et al. (2001). It was discovered in a search for an optical counterpart to the point-like X-ray source CXOU J085201.4461753 , although it has now been rejected as such. It is offset by about 2.' 4 from the nominal X-ray position.

We have also looked for an infrared counterpart in images from the Two Micron All Sky Survey (2MASS), the MSX6C Infrared Point Source Catalog (Egan et al. 1997), and the IRAS Sky Survey Atlas in the bands $1.25(\mathrm{~J}), 1.65(\mathrm{H}), 2.17(\mathrm{~K}), 8.28$ (A), 12.13 (C), 14.65 (D), 21.3 (E), 12, 25, 60, and $100 \mu \mathrm{m}$. In all three surveys, an unresolved source is found at the position of Source 18. The infrared sources included in the MSX and IRAS catalogues are listed in Table 2 as numbers 8 and 9 and are plotted as crosses in Fig. 2.

\section{Discussion}

\subsection{The nature of Source 18}

Source 18 is a resolved source in the field with the appearance of a small, elliptical nebula. In spite of its proximity to CXOU J085201.4-461753, their relationship is not clear. The area subtended by Source 18 at $20 \mathrm{~cm}$, after beam deconvolution, marginally encloses CXOU J085201.4-461753, which lies $26^{\prime \prime}$ away from the radio emission peak. At $13 \mathrm{~cm}$, CXOU J085201.4-461753 is well separated from the radio emission. The spectral index derived for Source 18 is strongly thermal, which makes it unlikely to be a PWN created by CXOU J085201.4-461753. The existence of infrared counterparts reinforces the interpretation of Source 18 as a thermal source.

Wright \& Barlow (1974) showed that an ionized, uniform, spherically symmetric mass loss flow leads to a spectrum with an index equal to 0.6 in the radio and infrared regimes. For example, the radio star P Cygni has a spectral index of 0.65 . Within the uncertainties, the spectral index derived for Source 18 is the same. Similar values have also been found for other radio stars. Therefore, Source 18 is probably an object with significant mass loss. The [12]-[25] and [25]-[60] color indices of this object, as derived from the fluxes listed for 


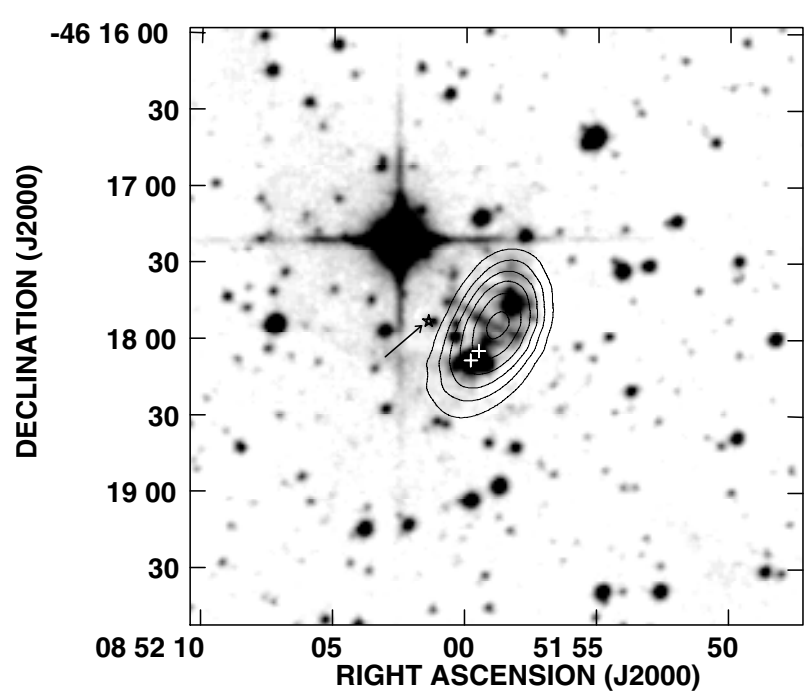

Fig. 4. Red ESO/R/MAMA image of the center of Vela Jr. The black star (pointed by an arrow) shows the position of CXOU J085201.4461753, while the two white crosses indicate the IR source IRAS 0852-4606 as given in the MSX and IRAS catalogues. A few radio contours at $13 \mathrm{~cm}$ are included, which correspond to Source 18 (see Table 1).

IRAS 08502-4606, are 0.12 and 0.27 respectively. These values place Source 18 in the region VIb of the van der Veen \& Habing (1988) two-color scheme, which corresponds to objects with separate hot and cold dust components indicating that the mass loss process has been significantly interrupted. van der Veen \& Habing (1988) conclude that objects in the VIb region are variable stars of which a small number have hot oxygen-rich material distributed separately from their cold dust component.

In optical images, Source 18 lies at the same location as Wray 16-30. Figure 4 shows the red ESO/R/MAMA image (downloaded from the Aladin Sky Atlas; Bonnarel et al. 2000), centered on Vela Jr, with radio continuum contours at $13 \mathrm{~cm}$ corresponding to Source 18 superimposed. The black star (pointed by an arrow) indicates the location of CXOU J085201.4-461753, while the white crosses correspond to the infrared peaks (according to the IRAS and MSX catalogues). In optical emission, Wray 16-30 appears complex, with two bright lobes symmetrically located on either side of a fainter linear filament with a NE-SW orientation. The whole structure is particularly bright in the $\mathrm{H} \alpha$ line (cf. Pellizzoni et al. 2002). The IR peaks lie on the brightest of the two lobes. The two lobes are enclosed within Source 18, with the jet-like axis of the optical nebula oriented orthogonally to the major axis of the radio nebula. The optical axis runs across the radio emission peak. We strongly believe that Source 18 is the radio counterpart of Wray 16-30. Higher resolution images would be very valuable to investigate the morphology at radio wavelengths in more detail.

The optical morphology of Wray 16-30 resembles that of "butterfly" planetary nebulae (e.g. Icke et al. 1999, and references therein). However, there is no agreement in the literature on the classification of Wray 16-30. As summarized in Sect. 3, references interpreting it as a peculiar PN or rejecting it as such are found almost equally. The problem arises from the absence of [O III] emission lines. For example, Stenholm \& Acker (1987) strictly reject as PNe those objects for which the ratio $I[\mathrm{O} \mathrm{III}] / I(\mathrm{H} \beta)=0$. Conversely, Kohoutek (2001) classifies them as PNe of excitation class 0 (corresponding to verylow-excitation objects, Sanduleak \& Stephenson 1973), provided additional indicative evidence is found. In Source 18, such evidence could be the elliptical morphology, the nonstellar size, and the thermal radio continuum spectrum, typical of the vast majority of PNe (although non-thermal spectra are seen in some cases; e.g. Isaacman 1981). The low excitation class contains objects which are evolving into typical $\mathrm{PNe}$ (Kohoutek 2001).

If Source 18 (or Wray 16-30) is indeed a PN, a physical association with CXOU J085201.4-461753 still cannot be ruled out. X-ray emission has been detected towards several "butterfly" PNe (Feibelman 1994; Kastner et al. 2003). Williams et al. (2004) found many X-ray sources strikingly close to PNe in M31. Although the X-rays are not coming from the PN themselves (they lie several arcseconds away), the authors conclude that there is a physical connection between them, and suggest that these associations may involve a PN and a low-mass X-ray binary occupying the same undetected star cluster. If this is the case, then CXOU J085201.4-461753 would not be the neutron star associated with Vela Jr, in accordance with the recent suggestion by Iyudin et al. (2005).

To further investigate a possible link between the PN and the X-ray source, we check to see if the distance to both sources are similar based on their extinction values. Landaberry et al. (2001) estimated the colour excess of Wray 16-30 to be $E(B-V)=0.93 \pm 0.45 \mathrm{mag}$. The reddening to $\mathrm{CXOU}$ J085201.4-461753 can be derived from the H I column density which, based on XMM-Newton observations, is $N_{\mathrm{H}}=$ (3.7-4.3) $\times 10^{21} \mathrm{~cm}^{-2}$ (Becker \& Aschenbach 2002). Using the ratio $N_{\mathrm{H}} / A_{J}=(5.6 \pm 0.4) \times 10^{21} \mathrm{~cm}^{-2} \mathrm{mag}^{-1}$ (Vuong et al. 2003) and the standard values $A_{V} / A_{J}=3.31$ and $R=$ $A_{V} / E(B-V)=3.1$ for diffuse dust (e.g. Mathis 1990), the colour excess of CXOU J085201.4-461753 turns out to be $0.76 \pm 0.12 \mathrm{mag}$. To apply the reddening-distance model of Chen et al. (1999), we first estimated the total reddening in the direction of CXOU J085201.4-461753 produced by the Galactic Plane along the line of sight to be 2.15 mag (Schlegel et al. 1998). The derived distances to Wray 16-30 and CXOU J085201.4-461753 are thus $3.0 \pm_{1.5}^{2.2} \mathrm{kpc}$ and $2.4 \pm 0.4 \mathrm{kpc}$ respectively. Therefore, considering the uncertainties, it is possible that both sources lie at the same distance.

\subsection{Is there a PWN associated with $C X O U$ J085201.4-461753?}

An alternative way to test if CXOU J085201.4-461753 is a neutron star is by searching for the radio counterpart corresponding to a PWN. Although no conclusions can be drawn from a negative result, a positive PWN detection is a strong indication that the powering source must be a neutron star.

Two radio features were suggested by Reynoso et al. (2004b) as a possible PWN blown by CXOU J085201.4461753. One of them was Source 18. From the discussion 
above, it is clear that this interpretation is unlikely. The other candidate was a $30^{\prime}$ long nebulosity. The new observations presented here reveal that this feature is, in fact, an artifact due to poor $u-v$ coverage in the interferometric radio data.

In Fig. 1, CXOU J085201.4-461753 lies on a local enhancement of diffuse emission. This region is not larger than a few arcmin in diameter and its emission never exceeds the $3 \sigma$ level, making it very difficult to establish its significance unambiguously. The integrated flux density of this feature using a boundary set rather subjectively is $76 \mathrm{mJy}$ at $20 \mathrm{~cm}$. If this feature were the PWN driven by CXOU J085201.4-461753, then it would be characterized by a flat non-thermal spectrum (see for example Giacani et al. 2001). Assuming a spectral index flatter than -0.3 , then the mean flux density at $13 \mathrm{~cm}$ over the same region (and with the same spatial filtering) should be $\gtrsim 4 \mathrm{mJy}_{\text {beam }}{ }^{-1}$. However, the measured flux density at this wavelength is only $\sim 1 \mathrm{mJy}$ beam $^{-1}$. Therefore, either the central emission at $20 \mathrm{~cm}$ is not a genuine source or it has a spectrum steeper than the expected model. In either case, no PWN is indicated.

\section{Conclusions}

We have made a high resolution, high sensitivity image of the center of the SNR Vela $\mathrm{Jr}$ at 13 and $20 \mathrm{~cm}$. The aim of the project was to detect a radio counterpart to the X-ray source CXOU J085201.4-461753, a neutron star candidate for the compact remnant of the SN explosion. There is no evidence for the existence of a PWN associated with CXOU J085201.4461753. The closest radio feature detected is a compact source of thermal origin, named Source 18 in this paper, which appears to be the counterpart of the optical source Wray 16-30 detected also at infrared wavelengths as IRAS 08502-4606. In spite of the lack of [O III] emission lines, we believe there is good evidence that Wray $16-30$ is a PN, probably belonging to the morphological class known as "butterfly" PNe. If so, it is also possible that CXOU J085201.4-461753 is physically associated with it. In that case, CXOU J085201.4-461753 would then not be the CCO of SNR Vela Jr. This interpretation is strengthened by the radio frequency results presented here, which do not indicate an association between CXOU J085201.4-461753 and Vela Jr.

The angular resolution of the present observations is insufficient to show whether Source 18 has an internal structure similar to the optical images. Higher resolution data could be obtained with the ATCA in an extended configuration and at a higher frequency.

A by-product from this study is the discovery of 30 new, uncatalogued unresolved sources, which may be useful for comparison with high energy searches. For almost half of these sources we were able to measure the flux densities at both frequencies, 1348 and $2368 \mathrm{MHz}$, and have computed spectral indices using the flux-flux method. The remaining sources were too weak at $13 \mathrm{~cm}$ to derive reliable results. Among those sources for which spectral indices could be measured, six were found to be extragalactic and five are clearly thermal, including the previously known source PMN J0853-4620. The only one with a spectral index close to 0.6, typical of stars with mass outflows, is Source 18.

Acknowledgements. The authors wish to thank the referee, Tyler Foster, for his very useful comments which helped to improve the quality of the paper. This project was partially financed by grants ANPCyT-14018 and UBACYT A055. During part of this work, E. M. R. was a visiting scholar at the University of Sydney. This research has made use of Aladin.

\section{References}

Acker, A., Cuisinier, F., Stenholm, B., \& Terzan, A. 1992, A\&A, 264, 217

Aharonian, F., Akhperjanian, A. G., Baze-Bachi, A. R., et al. 2005, A\&A, 437, L7

Allen, D. A., \& Glass, I. S. 1975, MNRAS, 170, 579

Aschenbach, B. 1998, Nature, 396, 141

Aschenbach, B., Iyudin, A. F., \& Schönfelder, V. 1999, A\&A, 350, 997

Becker, W., \& Aschenbach, B. M. 2002, Neutron Stars, Pulsars, and Supernova Remnants, ed. W. Becker, H. Lesch, \& J. Trümper (Garching: MPE), 64

Bonnarel, F., Fernique, P., Bienaymé, O., et al. 2000, A\&AS, 143, 33

Brazier, K. T. S., \& Johnston, S. 1999, MNRAS, 305, 671

Chatterjee, S., \& Cordes, J. M. 2002, ApJ, 575, 407

Chen, B., Figueras, F., Torra, J., et al. 1999, A\&A, 352, 459

Combi, J. A., Romero, G. E., \& Benaglia, P. 1999, ApJ, 519, L177

Costain, C. H. 1960, MNRAS, 120, 248

Duncan, A. R., \& Green, D. A. 2000, A\&A, 364, 732

Duncan, A. R., Stewart, R. T., Haynes, R. F., \& Jones, K. L. 1995, MNRAS, 277, 36

Egan, M. P., et al. 2003, The Midcourse Space Experiment Point Source Catalog, Version 2.3, Explanatory Guide (AFRL-VS-TR3004-1589)

Feibelman, W. A. 1994, PASP, 106, 756

Frail, D. A. 1998, in The Many Faces of Neutron Stars, ed. R. Buccheri, J. van Paradijs, \& M. A. Alpar (Dordrecht: Kluwer), NATO ASI Ser. C, 515, 179

Frater, R. H., Brooks, J. W., \& Whiteoak, J. B. 1992, Electrical Electron. Eng. Australia, 12, 103

Gaensler, B. M. 2004, in Young Neutron Stars and their environments, ed. F. Camilo, \& B. M. Gaensler (San Francisco: ASP), IAU Symp., 218, 151

Geppert, U., Page, D., \& Zannias, T. 1999, A\&A, 345, 847

Giacani, E. B., Frail, D. A., et al. 2001, AJ, 121, 3133

Giacani, E., Reynoso, E., Dubner, G., et al. 2005, AdSpR, 35, 1070

Green, A. J., Cram, L. E., Large, M. I., \& Ye, T. 1999, ApJS, 122, 207

Icke, V., Preston, H. L., \& Balick, B. 1999, AJ, 97, 462

Isaacman, R. 1981, A\&AS, 43, 405

Iyudin, A. F., Schonfelder, V., Bennett, K., et al. 1998, Nature, 396, 142

Iyudin, A. F., Aschenbach, B., Becker, W., Dennerl, K., \& Haberl, F. 2005, A\&A, 429, 225

Kastner, J. H., Balick, B., Blackman, E. G., et al. 2003, ApJ, 591, L37

Kohoutek, L. 2001, A\&A, 378, 843

Landaberry, S. J. C., Pereira, C. B., \& Araújo, F. X. 2001, A\&A, 376, 917

Mathis, J. S. 1990, ARA\&A, 28, 37

Mereghetti, S. 2001, ApJ, 548, L213

Pavlov, G. G., Sanwal, D., Kiziltan, B., \& Garmire, G. P. 2001, ApJ, 559, L131

Pellizzoni, A., Mereghetti, S., \& De Luca, A. 2002, A\&A, 393, L65 
Perryman, M. A. C., Lindegren, L., Kovalevsky, J., et al. 1997, A\&A, 323, L49

Reynoso, E. M., Green, A. J., Johnston, S., et al. 2003, MNRAS, 345, 671

Reynoso, E. M., Green, A. J., Johnston, S., et al. 2004a, PASA, 21, 82

Reynoso, E. M., Johnston, S., Green, A. J., et al. 2004b, in Young Neutron Stars and Their Environments, ed. F. Camilo, \& B. M. Gaensler (San Francisco: ASP), IAU Symp., 218, 277

Sanduleak, N., \& Stephenson, C. B. 1973, ApJ, 185, 899

Sault, R. J., Teuben, P. J., \& Wright, M. C. H. 1995, in Astronomical Data Analysis Software and Systems IV, ed. R. A. Shaw, H. E. Payne, \& J. J. E. Hayes (San Francisco: ASP), ASP Conf. Ser., 77,433

Schlegel, D. J., Finkbeiner, D. P., \& Davis, M. 1998, ApJ, 500, 525

Slane, P., Hughes, J. P., Edgar, R. J., et al. 2001, ApJ, 548, 814
Stenholm, B., \& Acker, A. 1987, A\&AS, 68, 51

Stupar, M., Filipovic, M. D., Jones, P. A., \& Parker, Q. A. 2005, AdSpR, 35, 1047

Turtle, A. J., Pugh, J. F., Kenderdine, S., \& Pauliny-Toth, I. I. K. 1962, MNRAS, 124, 297

van der Veen, W. E. C. J., \& Habing, H. J. 1988, A\&A, 194, 125

Velghe, A. G. 1957, ApJ, 126, 302

Vuong, M. H., Montmerle, T., Grosso, N., et al. 2003, A\&A, 408, 581

Williams, B. F., García, M. R., McClintock, J. E., \& Kong, A. K. H. 2004, ApJ, 128, 1588

Wray, J. D. 1966, Ph.D. Thesis Northwestern University, Volume 2706, Section B, 1710

Wright, A. E., \& Barlow, M. J. 1974, MNRAS, 170, 41

Wright, A. E., Griffith, M. R., Burke, B. F., \& Ekers, R. D. 1994, ApJS, 91,111 